\title{
Análisis de la convivencia escolar desde la perspectiva psicológica, legal y pedagógica en Colombia
}

\section{Analysis of school coexistence from the psychological, legal and pedagogical perspective in Colombia}

DOI: http://dx.doi.org/10.17981/cultedusoc.8.2.2017.06

Karen Elena Salas Viloria ${ }^{1}$, Harold Arturo Cómbita Niño². Corporación Universidad de la Costa, CUC.

\begin{abstract}
Resumen
La Convivencia Escolar en Colombia se aborda bajo las directrices de un marco legal y pedagógico en el que se evidencia la necesidad de propiciar una mayor articulación de los aportes de la Psicología. El artículo desarrolla un análisis crítico derivado de los principales lineamientos normativos en Colombia para la intervención pedagógica en convivencia escolar, tales como la Constitución Política (1.991), la Ley 115 (1.994) y la Ley 1620 (2013); así como los documentos oficiales pertenecientes al marco pedagógico que se utilizan en la orientación de los establecimientos educativos para el desarrollo de ambientes de aprendizajes que favorecen la institucionalización de las competencias ciudadanas. Se propone integrar los aportes científicos desde la Psicología en consistencia con las directrices legales y pedagógicas para posibilitar un abordaje con mayor interdisciplinariedad de la convivencia escolar en Colombia que favorezca la educación para la paz.
\end{abstract}

Palabras clave: Rol familiar, relaciones entre pares, acoso, relación padres-profesores, relación profesor-alumno.

Recibido: Octubre 12 de 2017 / Aceptado: Noviembre 30 de 2017.

\footnotetext{
${ }^{1}$ Psicóloga, Maestrante en Educación. Profesora Departamento de Psicología de las Interacciones Sociales. Universidad de la Costa. Correspondencia: ksalas@cuc.edu.co

${ }^{2}$ Magister en Gobierno de Tecnología Informática. Docente investigador Programa de Ingeniería de Sistemas. Universidad de la Costa. Correspondencia: hcombita1@cuc.edu.co
} 


\begin{abstract}
School Coexistence in Colombia is addressed under the guidelines of a legal and pedagogical framework in which the need to promote a greater articulation of the contributions of Psychology is evidenced. The article develops a critical analysis derived from the main normative guidelines in Colombia for the pedagogical intervention in school coexistence, such as the Political Constitution $(1,991)$, Law $115(1,994)$ and Law 1620 (2013); as well as the official documents pertaining to the pedagogical framework that are used in the orientation of educational establishments for the development of learning environments that favor the institutionalization of citizen competencies. It is proposed to integrate the scientific contributions from the Psychology in consistency with the legal and pedagogical guidelines to enable an approach with greater interdisciplinarity of the school coexistence in Colombia that favors education for peace.
\end{abstract}

Keywords: Family role, peer relations, harassment, parent-teacher relationship, teacher-student relationship.

\title{
Introducción
}

Los problemas derivados de la convivencia escolar tienen efectos que perjudican a los principales actores de la comunidad educativa. Las víctimas tienen una mayor predisposición a desarrollar trastornos de ansiedad, psicosomáticos o emocionales y en situaciones extremas se reportan casos de suicidio; por su parte, los victimarios presentan dificultades para establecer relaciones positivas con sus pares o figuras de autoridad, y son más propensos al desarrollo de conductas delictivas (Tresgallo, 2008; Olweus, 2006).

Los establecimientos educativos por su parte, afrontan como institución los problemas comunes tanto para víctimas como victimarios, es decir, disminución del rendimiento académico y disciplinario (Loredo-Abdalá, Perea-Martínez y LópezNavarrete, 2008).

Lo anterior, converge con la problemática de la deserción estudiantil, ya sea porque las víctimas quieren evitar ir a la escuela a ser maltratados o porque los victimarios a causa de su indisciplina les es cancelada la matrícula. Por lo anterior, al truncarse el proceso educativo de formación para la sociedad, se limitan las oportunidades para acceder a una mejor calidad de vida en su futuro; sin embargo, en el caso de los menores agresores que desertan el problema es aún mayor puesto que además se incrementan las posibilidades de desarrollarse en conductas delictivas, por lo tanto, para ellos permanecer en la escuela es un factor protector. (Prieto y Carrillo, 2009; Ramos de Fernández \& Sepúlveda, 2017)

Los problemas derivados de la convivencia escolar tienen efectos que exceden el ámbito individual de los directamente involucrados como víctimas y victimarios, y trasciende generando otra serie de problemáticas a nivel institucional, familiar, jurídico y social. Esto sumado a los altos índices de violencia escolar en el país, fundamentaron la necesidad de establecer en Colombia una serie de lineamientos legales a través de la expedición de leyes y códigos que las reglamentan; así como lineamientos pedagógicos a través de documentos guías del Ministerio de Educación Nacional 
$(\mathrm{MEN})$, para orientar a los actores de la comunidad responsables de la educación integral para la paz de los niños, niñas y adolescentes (Agencia de Prensa, 2012).

Acorde con la Organización Panamericana de la Salud (2013), Díaz-Aguado, (2005) y Ortega, (2000), la solución para la problemática de la violencia está en desarrollar estrategias validadas científicamente para la prevención de la misma. En ése sentido se hace fundamental el desarrollo de programas pedagógicos para la convivencia fortalecidos con un abordaje interdisciplinar; sin embargo, el Enfoque de Derechos, el cual es el oficialmente trabajado en Colombia, (MEN, 2011) si bien es fundamental y necesario en el ámbito jurídico, no brinda a los establecimientos educativos las suficientes herramientas y directrices que se necesitan para intervenir eficazmente en la construcción de relaciones sociales pacíficas.

Si se considera que "Las causas que obran para que una persona sea violenta pueden ser variadas, pero usualmente se asocian con variables de índole psicológica o social." (ENDS, 2010), se hace indispensable una mayor inclusión de la dimensión psicológica para aportar lineamientos a la comunidad educativa que posibiliten mayor eficacia de la promoción de la convivencia, así como el control y prevención de las problemáticas derivadas del inadecuado manejo de esta, como el acoso escolar.

Guardando consistencia con las directrices legales y pedagógicas para el abordaje de la convivencia en Colombia, se sugiere enriquecer la interdisciplinariedad de dicho abordaje desde los aportes científicos de la Psicología con el propósito de mejorar los procesos inherentes a la comprensión y desarrollo de las competencias ciudadanas, así como el fortalecimiento del desarrollo de habilidades, recursos y estrategias de afrontamiento en la comunidad educativa que favorezcan la educación integral para la paz.

\section{Análisis Crítico principales Políticas Públicas.}

\section{- Constitución Política de Colombia de 1.991}

La Constitución Política de Colombia se caracteriza por sus principios democráticos, participativos y pluralistas, los cuales se ven reflejados transversalmente a lo largo de toda la Carta Magna y que parten desde el preámbulo de la misma en la cual se establece que:

"en ejercicio de su poder soberano, representado por sus delegatarios a la Asamblea Nacional Constituyente, invocando la protección de Dios, y con el fin de fortalecer la unidad de la Nación y asegurar a sus integrantes la vida, la convivencia, el trabajo, la justicia, la igualdad, el conocimiento, la libertad y la paz, dentro de un marco jurídico, democrático y participativo que garantice un orden político, económico y social justo, y comprometido a impulsar la integración de la comunidad latinoamericana, decreta, sanciona y promulga la siguiente" (1991).

En este orden de ideas, uno de los elementos centrales de la Constitución en la construcción de Nación se confiere al Título II, el cual está dedicado a los Derechos Fundamentales. 
Es importante señalar que importantes artículos constitucionales, como por ejemplo los que se clasifican dentro de los derechos, garantías y deberes de los colombianos se ven afectados en los distintos escenarios y formas en las que se puede presentar la violencia en la cotidianidad de la vida social y comunitaria; pero particularmente en la cotidianidad de la escuela, dado fenómenos sociales como el acoso escolar.

Algunos de esos derechos que se ven afectados con la violencia escolar son: el derecho a la vida (Art. 11), a no ser sometido a tratos o penas crueles, inhumanos o degradantes (Art. 12), la protección y defensa de las personas que son abusadas o maltratadas por su condición económica, física o mental (Art. 13), el libre desarrollo de la personalidad (Art. 16), el no ser molestado por razón de las convicciones o creencias (Art. 18), el derecho a la honra (Art. 21), y sobre todo se vulnera el derecho a la Paz que es establecida como un derecho y un deber de obligatorio cumplimiento (Art.22).

Por todo lo anterior, se precisa la necesidad del Estado en la formulación de políticas sociales que se encaminen para promover la disminución del impacto social de las problemáticas derivadas de la violencia escolar y fortalezcan la formación en ciudadanía. En este sentido, se establecen acertadamente responsabilidades para lograr el cumplimiento de los derechos fundamentales de los niños (Art. 44) y de los adolescentes quienes deben ser protegidos y formados integralmente (Art. 45).

Para garantizar esto el Estado reconoce primeramente que la familia es el núcleo fundamental de la sociedad y que tiene la responsabilidad de sostener y educar a los niños (as) y adolescentes (Art.42); así mismo, se instituye a la educación como un derecho de la persona y un servicio público con una función social que formará al colombiano en el respeto a los derechos humanos, la paz y democracia, y que estará bajo la responsabilidad del Estado, la sociedad y la familia, otorgándose con obligatoriedad entre los 5 y 15 años de edad (Art. 67).

\section{- Ley 115 General de Educación}

La ley 115 es un instrumento para la organización y reglamentación del servicio educativo por parte de los establecimientos públicos y privados que lo suministran, en este sentido, se presentarán algunos de los elementos más significativos de la ley en relación al tema de la convivencia escolar.

Para empezar, la conceptualización adoptada de educación, la entiende como un proceso de formación permanente, personal, cultural y social fundamentado en una concepción integral de la persona humana, de su dignidad, de sus derechos y deberes, y en donde se resalta la función social de la educación la cual debe estar acorde con las necesidades e intereses de las personas, la familia y la sociedad (Art.1).

La educación por lo tanto, cumple una función socializadora en la que a través de un proceso de enseñanza y aprendizaje se forma a las personas para cumplir con las exigencias y expectativas en base al cumplimiento de los roles que de ellos espera la familia y la sociedad, a la vez que también busca el mejoramiento de dicha familia 
y sociedad mediante la formación en valores y derechos humanos que hagan posible la construcción de una realidad social pacífica, libre e incluyente.

Por su parte a los principales agentes socializadores, es decir, la familia y la escuela, se les delimitan una serie de responsabilidades a fin de dar cumplimiento a la función social de la educación.

La familia, es reivindicada nuevamente como el núcleo fundamental de la sociedad y como la primera responsable en la educación de los hijos. Se le demanda puntualmente a cumplir con ciertos deberes, dentro de los que cabe destacar algunos como el buscar y recibir orientación sobre la educación de los hijos (d) y educarlos proporcionándoles en el hogar un ambiente adecuado para su desarrollo integral (g). (Art.7).

La escuela es orientada a que cumpla unos objetivos comunes en todos sus niveles de formación que tienen como fin último lograr el desarrollo integral de los educados, para esto se definen varios compromisos como por ejemplo el implementar acciones para formar la personalidad y la capacidad de asumir con responsabilidad y autonomía los derechos y deberes (a), proporcionar una sólida formación ética y moral, y fomentar la práctica del respeto a los derechos humanos (b), desarrollar acciones de orientación escolar, profesional y ocupacional (f), entre otras. (Art.13); además, es de enseñanza obligatoria la educación para la justicia, la paz, la democracia, la solidaridad, la confraternidad, el cooperativismo y en general la formación en los valores humanos (d) (Art.14), así mismo se hace mención específica a la formación en los valores propios de la nacionalidad colombiana, los cuales son la solidaridad, la tolerancia, la democracia, la justicia, la convivencia social, la cooperación y la ayuda mutua. (Art. 20. D).

$\mathrm{El}$ educando es el centro del proceso educativo y debe participar activamente en su propia formación integral (Art.91). La formación del educando debe favorecer su pleno desarrollo de la personalidad, formación cultural, científica, técnica y en valores, por lo cual, los establecimientos educativos incluirán en el PEI acciones pedagógicas que favorezcan en los educandos en especial las capacidades para la toma de decisiones, adquisición de criterios, trabajo en equipo, administración eficiente del tiempo, asunción de responsabilidades, solución de conflictos y problemas, habilidades para la comunicación, la negociación y participación. (Art. 92).

Por su parte el educador, como orientador en los establecimientos educativos recibirá una capacitación y actualización profesional (a).Art. 104; sin embargo el mejoramiento profesional estará a cargo no solo de la nación, las entidades territoriales y las instituciones educativas, sino también de los propios educadores (Art. 110).

Es de resaltar, que si bien están claramente delimitadas las funciones de los actores de la comunidad educativa para favorecer la educación como un fin social y que propicie la formación integral del estudiante, la familia que se establece en la misma ley como la primera responsable de la educación y como núcleo fundamental de la sociedad, más allá de designarle las funciones que ésta debe cumplir, no se desarrolla sobre ella como tal ningún capítulo que establezca una normatividad a través de la cual se pueda ejercer un mecanismo de control para hacer cumplir a la familia con su deber como la principal agente de formación educativa. 
En otras palabras, se establece la normatividad sobre lo que debe cumplir la escuela para responder por las responsabilidades que se le asignan como establecimiento prestador de un servicio educativo, pero no se hace lo mismo con la familia. A la familia solo se le designan funciones que no se articulan con una normatividad que viabilice y garantice el cumplimiento de dichas responsabilidades asignadas. Por ejemplo, se trabaja por hacer cumplir o por lo menos exigir el cumplimiento del deber de un docente que esté presentando inadecuadas prácticas pedagógicas; pero ¿Qué se hace con respecto a un padre de familia que no brinde un adecuado acompañamiento a su hijo en su proceso de enseñanza - aprendizaje?

Desde la instancia de las políticas públicas a nivel educativo empieza a apreciarse a partir de este momento, la desarticulación entre escuela y familia, en donde aunque por ley ambas tienen como deber la formación integral de los niños/as y adolescentes, en la práctica no existe una normatividad clara dirigida a la familia para que cumpla con dicha función. A nivel educativo, la vigilancia del Estado recae fundamentalmente para la escuela, pero ¿Quién vigila a la familia en el cumplimiento de su rol en la educación?

\section{- Ley 1620 de 2013}

La normatividad en Colombia dando continuidad a sus avances para hacer frente a la problemática ocasionada por la violencia escolar, oficializa el 15 de Marzo de 2013 la publicación de la Ley 1620 que reglamenta el sistema nacional de convivencia escolar y formación para el ejercicio de los derechos humanos, la educación para la sexualidad y la prevención y mitigación de la violencia escolar.

En esta ley se plantea la necesidad de establecer las responsabilidades, protocolos y rutas de acción que deben cumplirse en los distintos niveles que conforman la estructura del Sistema nacional de convivencia escolar.

Es de resaltar de dicha ley que logra un muy importante avance para la regulación normativa en cuanto a convivencia, pues se presentan con mucha claridad las diferentes responsabilidades establecidas en la estructura del Sistema nacional de convivencia escolar que se constituye por instancias en tres niveles que son: el nacional, territorial y escolar. Es así, como en el nivel escolar se determinan claramente las responsabilidades del comité escolar de convivencia (Art. 13), responsabilidades de los establecimientos educativos (art. 17), responsabilidades del director o rector (art. 18), responsabilidades de los docentes (art. 19), responsabilidad de diseñar proyectos pedagógicos (art. 20), y responsabilidades de la familia (art. 22).

Complementario a lo anterior, se proporcionan los procesos y protocolos que se deben seguir en los casos en que se presente violencia escolar, con base a lo definido en La Ruta de Atención Integral para la Convivencia Escolar. (Art. 29), sobre la cual se establecen unos componentes que son: promoción (centrado en el desarrollo de competencias ciudadanas y la definición de los criterios de convivencia, así como de los mecanismos e instancias de participación); prevención (centrado en la formación integral del menor y en las causas de la misma por el contexto económico, social , 
cultural y especialmente el familiar) y Atención (estrategias que permitan asistir o abordar a cualquier miembro de la comunidad educativa en las situaciones en las que se presenten casos de violencia). (Art. 30).

Finalmente, se oficializa un protocolo de acción inicia con la identificación de las situaciones en donde hay casos de violencia escolar, los cuales serán remitidos al comité de convivencia escolar que lo tratará en base al manual de convivencia. El Comité de Convivencia es el encargado de poner el hecho en conocimiento de los miembros de la comunidad educativa involucrada y de los generadores de los hechos violentos, juntos buscarán primeramente conciliar garantizando el debido proceso y la atención integral y seguimiento al respectivo caso. Las situaciones de violencia escolar que por su gravedad no puedan ser atendidas en base al manual de convivencia, tendrán que ser reportadas por parte del Rector a la entidad estatal que corresponda según el caso. (Art. 31).

Con base a lo anterior, se puede establecer que el Protocolo de la Ruta de Atención Integral para la Convivencia Escolar trabaja el componente de atención pero no delimita procedimientos para el abordaje de la promoción y prevención de la violencia escolar, los cuales también hacen parte de la ruta de atención integral y que son indispensables para buscar una solución que nos lleve a asumir las problemáticas derivadas de una inadecuada convivencia escolar no solo desde la sanción y la norma sino desde la educación, la cual es el componente que posibilita la formación en ciudadanía. (Funes, 2017; Gregori \& Menéndez, 2017).

El protocolo de la Ruta de Atención Integral es más que todo un proceso administrativo para proceder bajo las normatividades de la escuela amparadas en las directrices legales con tal de garantizar el debido proceso en los casos que se presenten.

\section{- Análisis Crítico de los Principales Programas Curriculares}

En el ámbito de la educación, la Constitución Política y las leyes son las encargadas de reglamentar las normas que deben cumplirse para guiar el proceso educativo, en éstas se define lo que se debe hacer en las diferentes instancias que hacen parte de la comunidad educativa pero NO determinan los mecanismos pedagógicos y curriculares que facilitan la puesta en marcha de las directrices normativas.

El Ministerio de Educación Nacional (MEN) es el encargado de dirigir los criterios pedagógicos de la prestación del servicio educativo, así como velar por la evaluación y calidad de la misma.

\section{- Estándares Básicos de Competencias Ciudadanas (2004)}

La formación en ciudadanía es un área que ha recibido poca atención para su desarrollo curricular, los esfuerzos en éste ámbito han estado dirigidos a los currículos de las ciencias básicas como matemáticas, naturales, y lenguaje. Los Estándares Básicos en Competencias Ciudadanas se constituyen como el primer currículo oficial en éste ámbito y en él se establecen las directrices para que éstas puedan desarro- 
llarse en base a los contenidos temáticos para trabajar dependiendo del nivel o grado en el que se encuentren los educandos.

En el 2004, se publicaron los Estándares Básicos de Competencias Ciudadanas desde la perspectiva de Derechos. Lo más importante de esta publicación, es que es la primera aproximación del gobierno nacional por construir un currículo referente a ésta temática. En el país el desarrollo curricular había estado enfatizado en las ciencias básicas y poco se había trabajado al respecto en cuanto al componente de la formación ciudadana.

En los Estándares Básicos de Competencias Ciudadanas se establece la noción de competencia asumida por el MEN (2004), la clasificación de las competencias ciudadanas y las directrices para desarrollarlas dependiendo del grado en el que se encuentre el estudiante; sin embargo, es importante fortalecer la división que se hace por grado para subsanar algunas falencias contenidas en el documento como por ejemplo, que en el grado de octavo no se especifica el desarrollo de ninguna competencia emocional.

Temáticas como las habilidades sociales y emocionales son de amplio estudio de la Psicología y necesarias para el desarrollo de la sana convivencia y el establecimiento de una educación para la paz. Por lo anterior, se hace necesario aplicar los componentes psicopedagógicos a los lineamientos curriculares, teniendo como base científica la etapa del desarrollo moral, cognitivo y psicosocial en el que se encuentran los estudiantes para desarrollar las competencias emocionales y sociales acordes a su edad.

\section{- Institucionalización de las Competencias Ciudadanas.}

En el año 2011, hubo un nuevo esfuerzo por parte del Ministerio de Educación Nacional cuyo objetivo era brindar orientaciones para la institucionalización de las competencias ciudadanas, es así como se publican unas cartillas para guiar éste proceso, la Cartilla 1 Brújula, y la Cartilla 2 Mapa, en las cuales se exponen cinco ambientes para el desarrollo de las competencias ciudadanas.

Ambas cartillas pertenecen al programa nacional de competencias ciudadanas, y en ellas se delimitan cinco ambientes que son: las Instancias de Participación, el aula de clases, el tiempo libre, los proyectos pedagógicos y la gestión institucional. En cada uno de estos cinco ambientes es fundamental realizar un trabajo de intervención porque fueron seleccionados estratégicamente por representar la cotidianidad de interacción que se da en los Establecimientos Educativos.

Se parte de la premisa que la ciudadanía no ocurre en un contexto específico sino en cada escenario de la vida cotidiana donde interactúan los educandos, por lo cual se hace necesario trabajar con todos los ambientes para facilitar la institucionalización de las competencias ciudadanas con el propósito de que éstas se desarrollen en contextos reales que impliquen su aplicación a nivel más pragmático y vivencial y no limitadas a un conocimiento teórico. 


\section{- Componentes de los Ambientes de las Competencias Ciudadanas}

A continuación se relacionan los cinco ambientes con los componentes de cada uno de ellos.

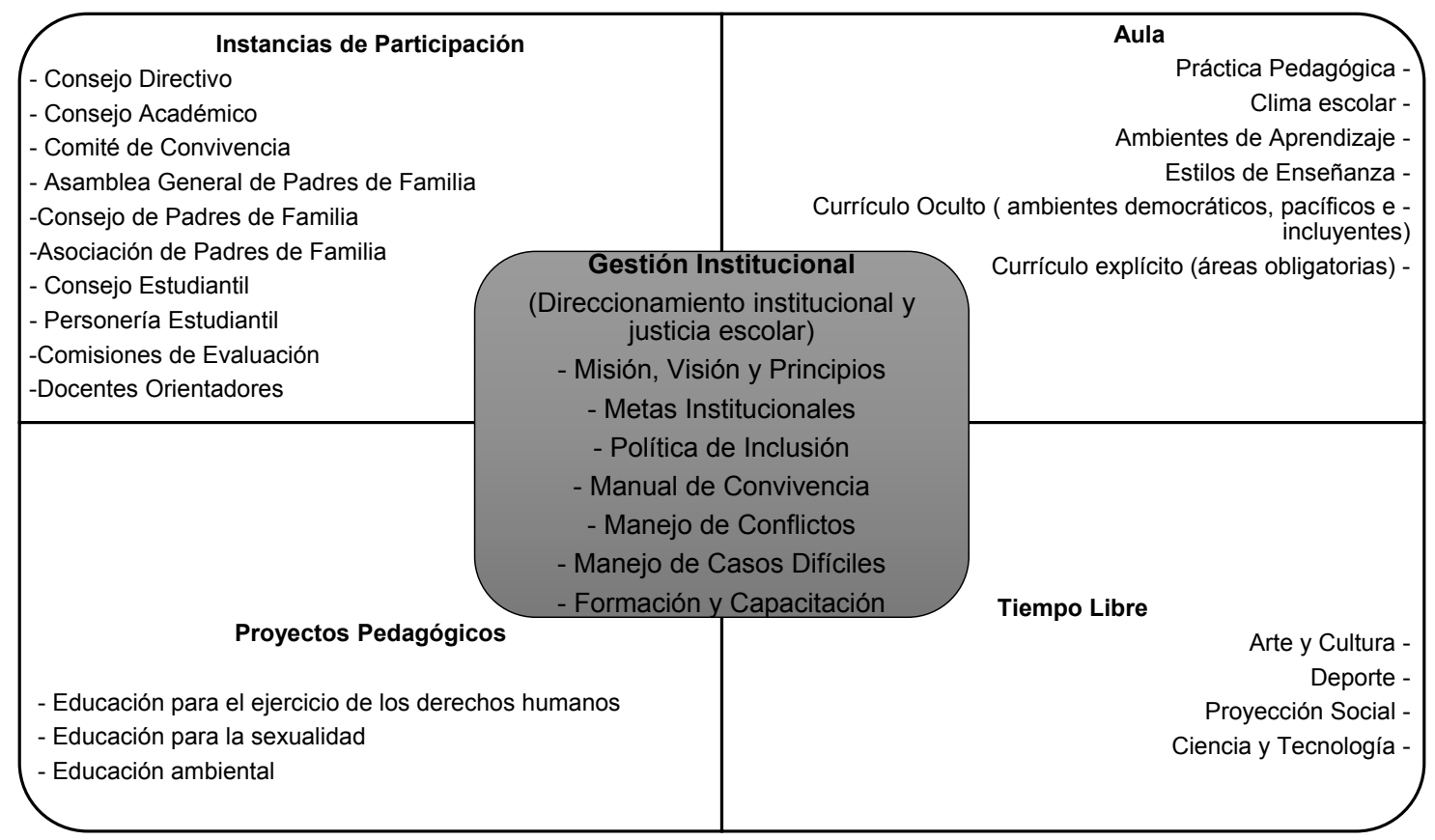

Figura 1. Escenarios de la Gestión Educativa orientados a la Convivencia Escolar

La Psicología es consistente con el marco legal colombiano, por lo que el diseño tanto de políticas públicas, desarrollos curriculares y proyectos pedagógicos, tienen que estar articulados con la comprensión de la conducta de las personas para que al implementarse sean más efectivos y ajustados a la realidad de la naturaleza del comportamiento humano.

El Modelo Ecológico de Bronfrenbrenner (1979) es pertinente para la visión del marco normativo y curricular de la nación colombiana puesto que permite la incorporación de los ambientes para el desarrollo de las competencias ciudadanas propuestos por el MEN; además permite la consideración de otros ambientes que otorgan una visión multidimensional que caracteriza la comprensión y abordaje de problemáticas con una naturaleza compleja como la de la convivencia. En otras palabras, el Modelo Ecológico de Bronfrenbrenner (1979) proporciona una teoría multifactorial que permite un abordaje multidimensional para una problemática con éstas características. Los conceptos básicos de la teoría ecológica son: Ontosistema: características propias del individuo; Microsistema: sistema de interacción más cercano al individuo; Mesosistema Interacciones de dos o más microsistemas en los que se desarrolla el individuo, Exosistema: Interacción entre dos o más sistemas en donde uno de ellos no incluye activamente al individuo; Macrosistema: Contenido 
cultural e ideológico de los micro, meso y exo sistemas; y Cronosistema: Época histórica del individuo (Bronfenbrenner, 1979).

En consecuencia, para lograr la institucionalización de las competencias ciudadanas en los diferentes miembros que conforman la comunidad educativa, es necesario agregar otros ambientes a los previamente establecidos en las directrices del MEN los cuales son: Instancias de Participación, el aula de clases, el tiempo libre, los proyectos pedagógicos y la gestión institucional; sin embargo, hay otros ambientes no contemplados como tal que hacen parte del proceso educativo en el que acontece la formación ciudadana, y que son necesarios para la comprensión, abordaje y desarrollo de la misma. Dichos ambientes serían: Ambiente Personal (Ontosistema), Ambiente Familiar (Microsistema), Ambiente Cultural (Exosistema) Ambiente Legal o Políticas Educativas (Macrosistema); visto como un sistema multidimensional con relaciones dinámicas e interdependientes que incide en el desarrollo de las competencias asociadas con la interacción social y la convivencia escolar (Castillo-Pulido, 2011; Avendaño-Villa, Cortés-Peña \& Guerrero-Cuentas, 2015).

Se considera importante resaltar la inclusión explícita del ambiente familiar, puesto que para lograr una sana convivencia se deben favorecer en el microsistema familiar condiciones específicas, como por ejemplo: estilos de crianza adecuados, la comprensión de los cambios que sufren los hijos, el desarrollo de recursos de apoyo a la familia, habilidades de comunicación y resolución de conflicto, promover una representación de la violencia que ayude a prevenirla. (Delgadillo Guzmán \& Argüello Zepeda, 2013).

\section{Discusión}

Se ha avanzado bastante a nivel normativo y curricular en el ámbito de la convivencia escolar, se han delineado políticas y orientaciones pedagógicas que promueven el cumplimiento de los derechos de la comunidad educativa; sin embargo, se hace necesario seguir trabajando en ése mejoramiento continuo, y para ello es importante reflexionar ¿Qué es lo que está fallando? ¿Las fallas son a nivel de las directrices del marco legal o curricular? ¿Falla el sistema educativo de la escuela o el sistema familiar? ¿Por qué si hay una ley que enmarca unos principios de convivencia, paz y respeto por los derechos humanos, esto no se hace efectivo en el ámbito escolar?

Las directrices que se promueven desde el Estado y la forma de operacionalizarse en la cotidianidad de la vida y la escuela, marcan una brecha entre la ley y la práctica. La escuela es un reflejo de la dinámica social, por lo tanto hasta que el proyecto de nación enunciado en la constitución política en sus leyes, no se constituyan en buenas prácticas cotidianas de convivencia y paz en todos sus niveles y estamentos, posiblemente la escuela seguirá siendo un lugar que refleje una sociedad disfuncional marcada por la solución de los conflictos por la vía violenta.

Fenómenos complejos como la convivencia requieren de un abordaje multidisciplinar que parta de una comprensión de sus múltiples dimensiones; no obstante, luego de revisar el marco legal y curricular de la convivencia en Colombia, se considera que uno de los elementos fundamentales para mejorar y analizar tiene que ver 
con el lugar dado a la familia en la educación, lugar que se reconoce en el papel a través de funciones claramente definidas desde el marco legal y pedagógico, pero no articuladas de manera clara en la práctica y los procesos de formación del educando.

Se hace por lo tanto necesario seguir trabajando en la integración de toda la comunidad educativa, especialmente en articular la participación de la familia (Cerezo Ramírez, 2011), quien acorde a lo establecido en el sistema educativo colombiano, debe reivindicársele en su posición de primera responsable de la educación de los menores (entendiendo educación desde su función social más que académica) y para ello han de delinearse y/o establecerse los mecanismos y/o procedimientos legales, normativos, jurídicos, curriculares y pedagógicos que regulen el cumplimiento de la misma.

Desde la Psicología y otros ámbitos del conocimiento científico dedicados al estudio del ser humano, sabemos que la familia, la escuela y la sociedad son los principales espacios de socialización del ser humano que pueden facilitar o inhibir el desarrollo, el bienestar, la salud mental y la calidad de la educación de los niños y jóvenes Smokowski, \& Holland, 2005; (Maganto \& Bartau, 2011; Cortés, 2015).

En la escuela y especialmente en la familia, el infante adquiere los primeros esquemas, vínculos afectivos (Bowlby, 2006) y los modelos del aprendizaje social (Bandura, 1987), en torno a los que estructurará su personalidad y relaciones sociales, estos a su vez influirán en el resto de su ciclo vital y las relaciones que establezcan en la vida adulta González-Pineda. \& Núñez (2005).

Por tal razón, la familia y la escuela son los principales espacios de socialización, sin embargo hoy en día la familia y la escuela, los dos sistemas tradicionales de educación, están disociados y es común encontrar que se profieran mutuamente la responsabilidad sobre la pérdida de valores y los altos índices de violencia que se están presentando en las escuelas. Este desencuentro entre sistema familiar y sistema educativo está haciendo que la labor de educar sea cada vez más compleja y utópica (Amar, Abello, Martínez, Monroy, Cortés \& Crespo, 2011).

En este sentido, es importante revalidar los aportes de la Psicología, la cual es una disciplina científica consistente con el marco legal y curricular colombiano para el ejercicio de los derechos humanos y la formación en ciudadanía, y que desde los conocimientos propios a su disciplina puede favorecer la consecución de la sana convivencia y la integración de la escuela y familia.

La historia en nuestro país nos muestra que el mal diseño de las políticas públicas radica en que muchas de las medidas adoptadas por los gobiernos no están alineados con la conducta natural de las personas y debido a esa falta de entendimiento del comportamiento humano, fracasan muchas de las directrices gubernamentales.

La Psicología es consistente con el marco legal colombiano, por lo que el diseño tanto de políticas públicas, desarrollos curriculares y proyectos pedagógicos, tienen que estar articulados con la comprensión de la conducta de las personas para que al implementarse sean más efectivos y ajustados a la realidad de la naturaleza del comportamiento humano. 


\section{Referencias}

Agencia de Prensa. (16 de Marzo de 2012). Colombia es uno de los Países con mayores cifras de Matoneo. Recuperado el 20 de agosto de 2014 de http://www.mineducacion.gov.co/observatorio/1722/article-300099.html

Amar, J. J., Abello, R., Martínez, M. B., Monroy, E., Cortés, O., \& Crespo, F. (2011). Social categorization and childhood cognition about poverty in children: An approach from the psychological essentialism. Investigación y Desarrollo, 19(1), 116139.

Avendaño-Villa, I., Cortés-Peña, O. F., \& Guerrero-Cuentas, H. (2015). Competencias sociales y tecnologías de la información y la comunicación como factores asociados al desempeño en estudiantes de básica primaria con experiencia de desplazamiento forzado. Diversitas, 11(1), 13-36.

Ballesteros de Valderrama, B. P., Cortés, O. F., \& Forero, D. (2001). Aplicación del modelo de ecuaciones estructurales en le explicación del comportamiento social infantil y juvenil en el estrato socio-económico bajo la ciudad de Bogotá. Suma psicol, 8(1), 1-54.

Bandura, A. (1987). Teoría del Aprendizaje Social. Madrid: Espasa-Calpe.

Bowlby, J. (2006). Los vínculos afectivos: formación, desarrollo y perdida (5ta Ed.). Madrid: Editorial Morata

Bronfenbrenner, U. (1979). The Ecology of Human Development: Experiments by Nature and Design Cambridge, MA: Harvard University Press.

Castillo-Pulido, L. E. (2011). El acoso escolar. De las causas, origen y manifestaciones a la pregunta por el sentido que le otorgan los actores. Magis. Revista Internacional de Investigación en Educación, 4(8).

Cerezo Ramírez, F. (2011). Políticas de convivencia escolar: percepción y eficacia desde la perspectiva familiar. REIFOP, 14(1), 313-323. Recuperado de: http//www. aufop.com

Constitución Política de Colombia. (1991). Preámbulo, Artículos 11, 12, 13, 16, 18, 21, $22,42,44,45,67$.

Delgadillo Guzmán, L. G., \& Argüello Zepeda, F. J. (2013). El bullying una manifestación de deterioro en la interacción social entre pares. Ra Ximhai, 9(3).

Díaz-Aguado, M.J. (2005). La violencia entre iguales en la adolescencia y su prevención desde la escuela. Psicothema, 17 (4), 549-558.

Funes, S. (2017). Resolución de conflictos en la escuela: una herramienta para la cultura de la paz y la convivencia. Contextos Educativos, 7(1), 91-106.

González-Pienda. J.A., \& Núñez, J.C. (2005). La implicación de los padres y su incidencia en el rendimiento de los hijos. Revista de Psicología y Educación, 1(1), $115-134$

Gregori, E. \& Menéndez, J. (2017). La participación de los estudiantes como evaluadores. Un estudio en las titulaciones universitarias de las artes. Perfiles educativos, 39(156), 141-158. 
Loredo-Abdalá, A., Perea-Martínez, A., \& López-Navarrete, G. E. (2008). Bullying”: acoso escolar. La violencia entre iguales. Problemática real en adolescentes. Acta pediátrica de México, 29(4), 210-214.

Maganto, J. M., \& Bartau, I. (2003). La formación de padres en el marco de las relaciones entre la familia, la escuela y la comunidad. La Formación de Padres un Modelo de actuación.

Ministerio de Educación Nacional de Colombia. (2011). Orientaciones Para la Institucionalización de las Competencias Ciudadanas, Cartilla 1, Brújula, Programa de Competencias Ciudadanas, Bogotá, Colombia.

Ministerio de Educación Nacional de Colombia. (2011) Orientaciones para la Institucionalización de las Competencias Ciudadanas. CARTILLA 2 Mapa. Programa de Competencias Ciudadanas. Bogotá, Colombia.

Ministerio de Educación Nacional de Colombia. (2004). Estándares Básicos de Competencias Ciudadanas. Bogotá, Colombia.

Ramos de Fernández, I., Sepúlveda Jara, J. \& Fernández Ramos, M. (2017). Physical and psychological profile of the adolescent victimary of Bullying. Archivos Venezolanos de Puericultura y Pediatría, 80(2), p. 53.

Peña, O. F. C. (2015). Well-Being Labor Teaching in the Educational Public Sector of Barranquilla-Colombia. Procedia-Social and Behavioral Sciences, 191, 28352843.

Prieto, Q. M. y Carrillo, N. J. (2009). Fracaso escolar y su vínculo con el maltrato entre alumnos: el aula como escenario de la vida afectiva. Revista Iberoamericana de Educación, 5(25), 1-8.

Profamilia (2010). Encuesta Nacional de Demografía y Salud - ENDS. Violencia Contra las Mujeres y los Niños. Recuperado el 20 de Agosto de 2016, de http:// www.profamilia.org.co/encuestas/Profamilia/Profamilia/index.php?option=com_content\& view $=$ article\&id $=146 \&$ Itemid $=116$

Olweus, D. (2006). Conductas de acoso amenaza entre escolares. Tercera edición. Madrid: Morata.

Ortega, R. (2000). Educar la convivencia para prevenir la violencia . En R. Ortega. Educar la convivencia para prevenir la violencia. Madrid: A Machado Libros, S.A.

Organización Panamericana de la Salud. (2013). Prevención de la violencia: la evidencia. El Paso, TX: OPS , Serie de orientaciones sobre prevención de la violencia: la evidencia. Disponible en: http://apps.who.int/iris/bitstream/10665/85671/1/9789275317488 spa.pdf

República de Colombia. (1994). Ley 115 de Febrero 8 de 1994. Artículos 1, 7, 13, 14, 20 , 91, 92, 104 y 110. Bogotá: Imprenta Nacional.

República de Colombia. (1994). Ley 1620 de 2013. Artículos 13, 17, 18, 19, 20, 22, 29, 30 y 31. Bogotá: Imprenta Nacional. 
Smokowski, P. \& Holland, K. (2005). Bullying in School: An Overview of Types, Effects, Family Characteristics, and Intervention Strategies. Children \& School, 27, 101-110.

Tresgallo Saiz, E. (2008). Violencia escolar ("Bullying”): documento para padres y educadores. Revista Española de Orientación y Psicopedagogía, 19(3), 331-336. Disponible en: http://www.redalyc.org/pdf/3382/338230780007.pdf 